\title{
HALLAZGOS HISTOMORFOMÉTRICOS DE LA PRÓSTATA RESIDUAL Y CELDA PROSTÁTICA TRAS LA CIRUGÍA RETROPÚBICA POR H.B.P.: (I) DATOS BASALES OBTENIDOS EN LOS ÚLTIMOS 70 CASOS CONSECUTIVOS
}

\author{
Juan José Ballesteros Sampol, José Royo Lázaro’ y Josep Lloreta Trull?.
}

Servicios de Urología, Radiología' y Patologíaª de los Hospitales del I.M.A.S. Barcelona. España.

\begin{abstract}
Resumen.- OBJETIVO: Exponer los datos que obtenidos en el estudio patológico de las piezas operatorias, así como las biopsias de la próstata residual y cápsula prostática que junto a los que nos proporcionan los métodos por imagen nos ofrecen la situación "basal" en que, tras la adenomectomía transcervical hemostática con vesicocapsuloplastia, queda la celda y la prostata residual periférica. Todo ello como primer paso para el estudio a largo término de su evolución y su posible implicación en la génesis de una nueva patología obstructiva o neoplásica.
\end{abstract}

MÉTODO: Lo constituyen las últimas 70 adenomectomías transcervicales (ATH) con la variante de vesicocapsuloplastia (VCP) aplicada recientemente y tras una experiencia anterior de 1.033 cirugías retropúbicas. Se analizan los hallazgos histológicos de las piezas operatorias, de las biopsias de glándula residual y de cápsula prostática y se constatan los cambios inmediatos que la cirugía ocasiona con medición de los volúmenes de próstata residual por ecografía. Como base comparativa se analizan próstatas residuales de piezas de prostatectomía o cistoprostatectomía radical en sujetos "jovenes".

RESULTADOS/CONCLUSIONES: Se obtienen datos objetivos sobre las lesiones histológicas dominantes y asociadas halladas en la pieza de adenomectomía. Igualmente sobre los mismos datos que ofrecen las biopsias de próstata residual periférica junto al volumen de dicha zona y que deberán, si nuestras suposiciones son correctas, establecer a medio- largo plazo criterios de aproximación pronóstica sobre la llamada reexpansión de la celda y el posible establecimiento de una neopatología obstructiva.

Palabras clave: HBP. Próstata residual. Reexpansión de celda.

Summary.- OBJECTIVES: To present the data obtained from surgical specimens and biopsies of residual prostate and prostatic capsule, which in addition to the information provided by imaging tests give us the baseline situation of the prostatic surgical bed and residual prostate after transcervical hemostatic adenomectomy with capsule plasty. All this is the first step for the longterm study of its evolution and possible implications in the genesis of new obstructive or neoplastic pathology.

METHODS: 70 consecutive cases of transcervical hemostatic adenomectomy with the variation of capsule 
plasty, recently developed, after a previous experience of 1033 retropubic surgeries.

We analyze the histologic findings in surgical specimens, biopsies of residual gland and prostate capsule, and confirmed the immediate changes surgery causes, measuring the volumes of residual prostate by ultrasound. As the base for comparisons we analyzed residual prostates from prostatectomies or radical cystoprostatectomy specimens in "young" patients

RESULTS/CONCLUSIONS: We obtained objective data about predominant and associated histological lesions found in adenomectomy specimens. In the same way, the same data were obtained from biopsies of peripheral residual prostate together with the volume of such zone, which should establish, if our hypothesis is right, the mid-long-term prognostic criteria for the approach to the so-called post operative reexpansion of the prostatic hollow and the possible establishment of new obstructive pathology.

Keywords: $B P H$. Residual prostate. Reexpansion of the prostatic hollow.

\section{INTRODUCCIÓN}

La génesis y progresiva evolución de la H.B.P. tiene como consecuencia la formación de una tumoración benigna de la zona transicional que en su crecimiento más o menos concéntrico tiene el doble efecto de incrementar el volumen de la glándula y de constreñir la luz de la uretra prostática. Cuando este segundo efecto se traduce en clínica urinaria con

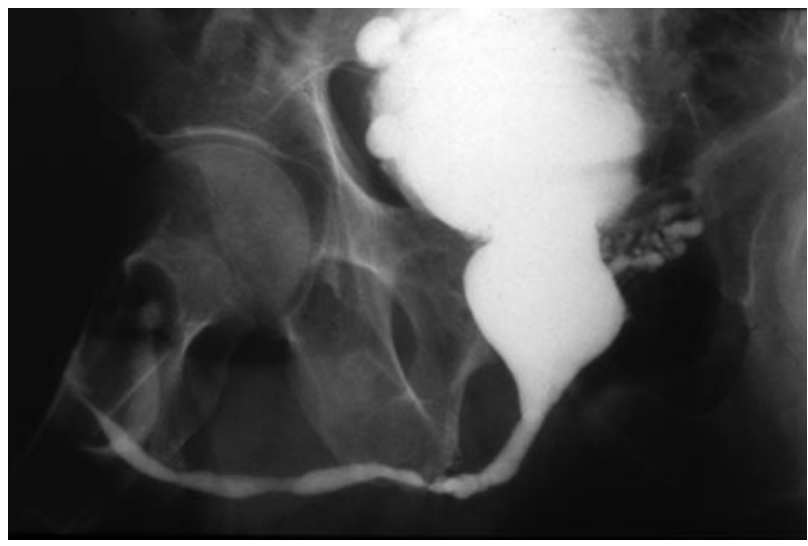

FIGURA 1. Amplia celda después de una cirugía retropúbica a los 3 años. criterios objetivos de obstrucción se decide la cirugía desobstructiva. En el momento actual son varias las opciones que se ofrecen para conseguirlo ya que, a las ya clásicas adenomectomía abierta y R.T.U. lo bipolar) se suman métodos menos invasivos como la TUNA y la "enucleación" con holmium-laser, entre otros de menor interés.

Tras una cirugía abierta indicada en glándulas de volumen igual o superior a los $65 \mathrm{cc}$ se van a producir dos hechos anatomohistológicos: la retracción de la celda prostática al cesar la "ocupación" por la HBP y la posibilidad de desarrollo histológico de la llamada próstata residual o periférica. El primero de ellos puede tener una intensidad variable de manera que es posible ver celdas muy amplias incluso varios años después de la cirugía (Figura 1), a la vez que tener tal intensidad que ocasione una retracción completa de la loge que reproduce un patrón obstructivo (Figura 2) que precisará solución quirúrgica, generalmente, endoscópica. El segundo efecto, la reexpansión, puede dar lugar a situaciones (Figura 3) que exijan desde el retratamiento médico de la neohiperplasia a la desobstrucción. Por fin y de mayor interés clínico, es la posibilidad de desarrollo neoplásico de dicha próstata residual. En este sentido, somos partidarios de seguimiento en CCEE de nuestro propio servicio de todos aquellos operados durante un período de 5 años. Pasado este tiempo son remitidos a asistencia "primaria" para que su médico de cabecera siga con controles de su PSA.

La retracción de la celda en la forma expresada anteriormente es rara tras cirugía abierta retropú-

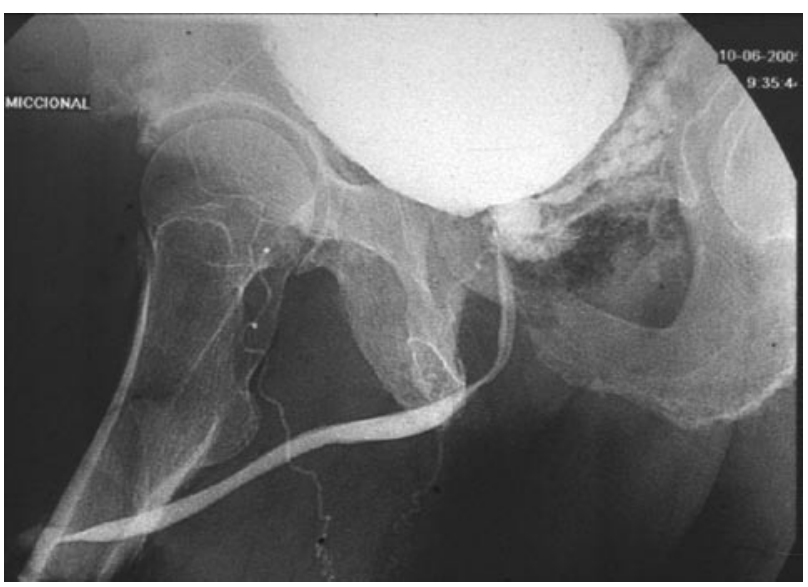

FIGURA 2. Intensísima retracción de la celda en un caso excepcional acaecida a los 4 meses de la enucleación de una glándula de 130cc. 


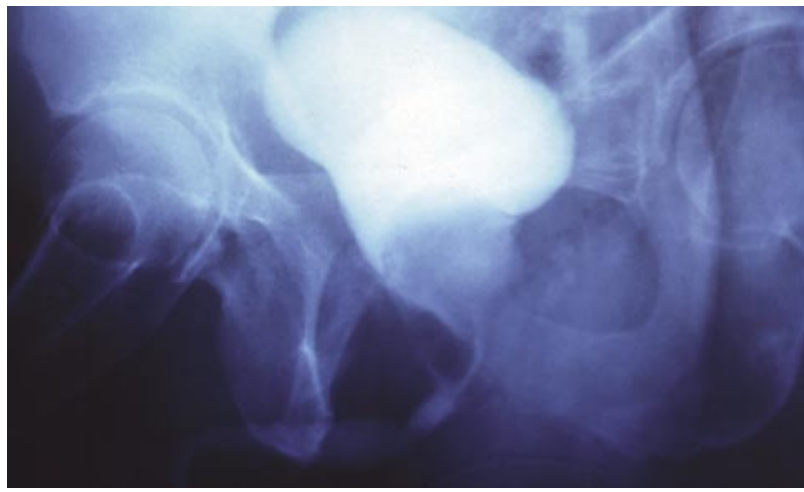

FIGURA 3. Reexpansión prostática hiperplásica a los 4 años de la cirugía.

bica. En nuestra experiencia y sobre 850 casos "valorables" se ha presentado en 8 , es decir un $0,94 \%$. Salvo un caso extraordinariamente precoz que se presentó a los 3 meses de la cirugía, los demás se han detectado entre los 12 y 48 meses. La solución aplicada ha sido siempre endoscópica mediante RTU bien aislada, bien combinada con Sachse previo o TCP (trigonocervicoprostatomía). Los hallazgos histológicos nunca han observado la presencia de tejido hiperplásico significativo siendo mayoritario el descubrimiento de tejido fibroso, fibras de músculo liso y prostatitis crónica.

En una ocasión se halló tejido neoplásico y, en dos, hubo asociación con litiasis múltiple vesical $y$, de celda, en reloj de arena. De interés nos parece también un caso desarrollado tras la enucleación de una próstata de volumen apreciable $(110 \mathrm{cc})$ y en la que se colocó surgicel hemostático en la celda comprimido por el globo de la sonda. La oxicelulosa puede ser la causante de reacción "fibrótica" que de un aspecto característico a la ecografía posterior (Figura 4).

La segunda posibilidad, es decir el crecimiento de una nueva patología hiperplásica capaz de reproducir la obstrucción, es un hecho que la progresión en la espectativa de vida del varón puede hacer más plausible de ahora en adelante. Esta circunstancia, en nuestra serie, se ha producido en 22 ocasiones $(2,6 \%)$. La solución en la mayoría ha sido la RTU pero en 5 casos se llegó a volúmenes considerables que aconsejaron la vía abierta. Como caso anecdótico podemos citar el de un paciente adenomectomizado a los 60 años (glándula de $60 \mathrm{grs}$ ), resecado por dos veces (22 y 20 grs respectivamente) y, nuevamente adenomectomizado por vía retropúbica (50 grs de HBP) en un periodo de 15 años. ¡En total 152 grs de HBP en 4 cirugías!.

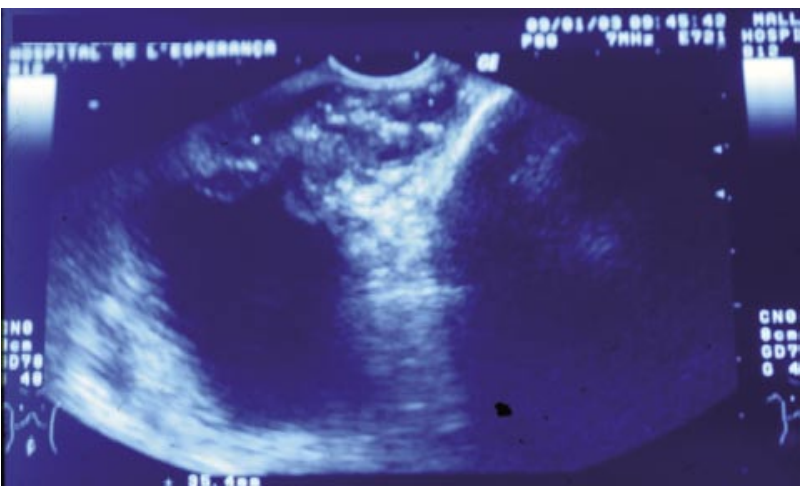

FIGURA 4. Ecografía transrectal a los 6 meses de la enucleación de una glándula de $110 c c$ y en la que se introdujo oxicelulosa con fines hemostáticos.

La idea que está generando este estudio va a ser la de observar qué casos y con qué histologías basales puede ser más probable que ocasionen estas neopatologías lo que, pensamos, puede ser importante cuando cada vez se da más importancia a los fenómenos inflamatorios en relación con HBP y Ca. de próstata $O$ se plantean problemas de interpretación de algunas lesiones de "atrofia" con las neoplásicas. Para ello nos ha parecido esencial conocer tales lesiones en el momento de la cirugía como patrón de base con el que comparar las que se hallarán en el seguimiento del protocolo establecido.

\section{MATERIAL Y MÉTODO}

Como ya adelantábamos lo constituyen los últimos 70 pacientes varones intervenidos por cirugía abierta siguiendo la técnica, ya publicada, de adenomectomía transcervical con vesicocapsuloplastia a la que remitimos al lector (1). Recordaremos sucintamente que se procede a la enucleación del "adeno$m a^{\prime \prime}$, a la obtención de dos biopsias de la glándula residual (una de cada lóbulo, por lo menos y dependiendo del volumen glandular) y a la resección de una franja de unos $1,5 \mathrm{cms}$ de anchura de la región cervicocapsular (Figura 5).

De dicha intervención obtendremos, por tanto, anatomía patológica de la "pieza", de las biopsias "residuales" y de la cápsula prostática.

Además, hemos obtenido datos de piezas de prostatectomías y cistoprostatectomías radicales de sujetos "jovenes" (50-55 años) en los que la HBP de la zona transicional debería ser poco desarrollada en relación a la zona periférica lo que nos permitió observar la histología antes de que la compresión de la HBP pudiera modificarla. 


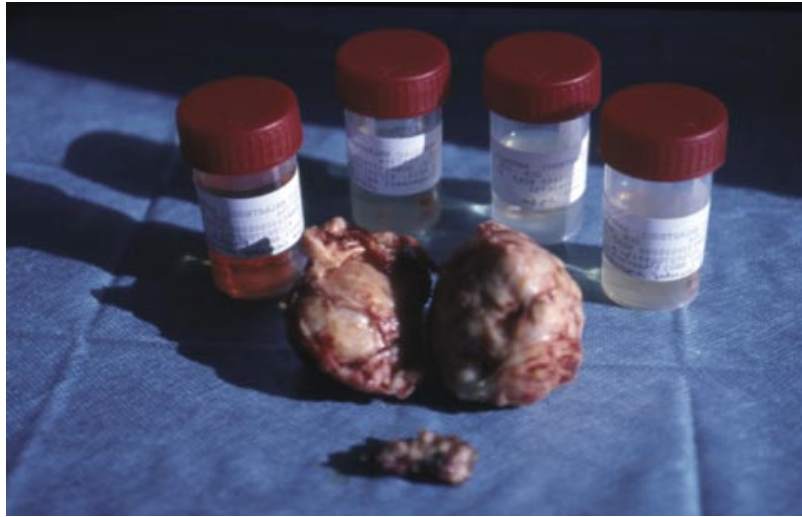

FIGURA 5. Especímenes extirpados: lóbulos prostáticos, fragmento capsulocervical y cuatro biopsias residuales.

a) Datos de la glándula enucleada: Han sido 70 piezas quirúrgicas con un peso medio de 65,35 grs (de 32 á 172 grs). Las lesiones, reflejadas en el Tabla l, las podemos dividir en lesión principal y lesiones asociadas. La lesión principal, en todos los casos, ha sido la hiperplasia, básicamente nodular (82\%) (Figura 6) y, en menor medida, glandular y adenomatosa. En un $18 \%$ de los casos dicha lesión ha sido considerada "pura" por la ausencia o mínima representación de lesiones asociadas. Estas, cuando significativas, han estado presentes en más del $80 \%$ de los casos

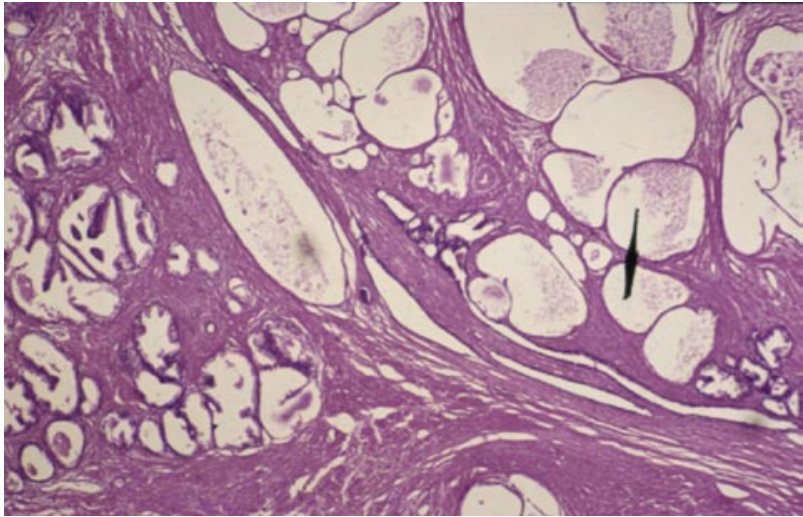

FIGURA 6. Histología habitual de las glándulas extirpadas. Hiperplasia nodular (40 x).

con una mayoría clara de lesiones inflamatorias, preferentemente crónicas (hasta un $71 \%$ ). De interés es el hallazgo de lesiones de cáncer prostático incidental $(6,25 \%)$ y PIN de alto grado $(3,1 \%)$. El resto de lesiones forman una miscelánea que incluye "atrofias", hiperplasia leiomiomatosa, metaplasia escamosa y microabscesos.

b) Datos de las biopsias de glándula residual: Quedan expresadas en el Tabla II y forman también una amplia miscelánea donde encontramos el mismo tipo

\section{A. LESIÓN PRINCIPAL}

Hiperplasia nodular $.82 \%$

Hiperplasia glandular. $14 \%$

Hiperplasia adenomatosa. $3 \%$

\section{B. LESIONES ASOCIADAS}

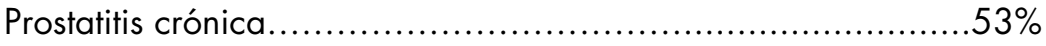

Formas agudas, mixtas y microabscesos............................. 18\%

Cáncer de próstata....................................................... 25\%

Focos de atrofia...................................................... $6,25 \%$

PIN de alto y bajo grado........................................... $3,1 \%$

Hiperplasia leiomiomatosa.......................................... $3,1 \%$

Metaplasia escamosa................................................. 


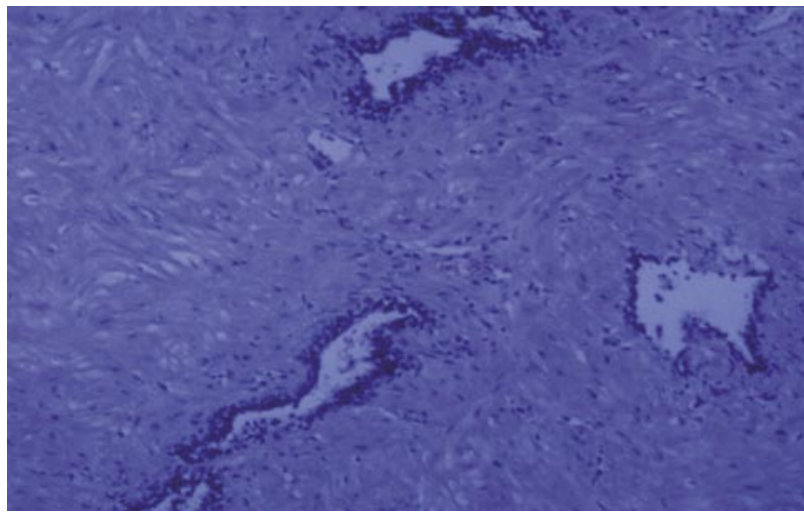

FIGURA 7. Biopsia residual: marcada atrofia. No se aprecian acinis glandulares y tan solo los conductos. Componente inflamatorio crónico asociado entre tejido estromal y musculatura lisa (200 x).

de lesiones pero con notable diferencia en sus porcentajes con relación a lo visto en la pieza operatoria. Así la representación de lesiones hiperplásicas sesitúa en torno al $21 \%$.

Por el contrario, hallazgos que representan la ausencia de tales lesiones, la existencia de "atrofias", inflamación crónica y aguda, tejido fibroso o fibroadiposo y pared de vesícula seminal triplican las lesiones hiperplásicas (Figuras 7 y 8). Un corolario simple invitaría a suponer que las biopsias que revelan tales hallazgos puedan ser de valor pronóstico cara a aventurar, con fundamento, la no reproducción de la lesión hiperplásica neoformada. Es evidente que el seguimiento y control de tales casos a

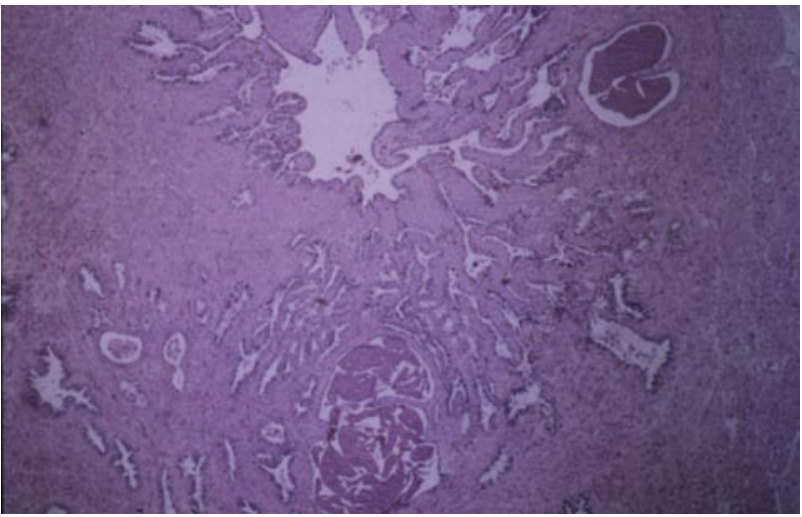

FIGURA 8. Biopsia residual: la práctica ausencia de zona periférica por el crecimiento de la hiperplasia hizo que esta biopsia interesara pared de vesícula seminal. (200 x).

medio-largo plazo nos confirmará o desmentirá tal hipótesis de trabajo.

c) Datos de la cápsula extirpada: El espesor de cápsula extirpada ha oscilado sobre la pieza en formol entre 6 y $16 \mathrm{~mm}$ lo que significa que "en fresco" nos hemos movido entre 1 y $2 \mathrm{cms}$. Aquí hay un predominio bastante acusado de los hallazgos (Tabla III). En un $66 \%$ de casos está constituida por músculo liso con fibrosis, un $17 \%$ es músculo liso puro, el $6 \%$ es fibrosis pura y, el resto, tejido fibroadiposo.

Puede extrañar el por qué de la resección de este segmento cervicocapsular y la razón no tiene que ver con la hipótesis de trabajo referida sino como

TABLA II. LESIONES OBTENIDAS EN LAS BIOPSIAS DE LA ZONA PERIFÉRICA TRAS LA ADENOMECTOMÍA.

\begin{tabular}{|l|c|}
\hline$\bullet$ Atrofia glandular & $27 \%$ \\
\hline$\bullet$ Hiperplasia glandular & $21,4 \%$ \\
\hline$\bullet$ Tejido fibromuscular estromal & $16,7 \%$ \\
\hline$\bullet$ Ausencia glandular+esclerosis+musculo liso & $14 \%$ \\
\hline$\bullet$ Prostatitis crónica & $6 \%$ \\
\hline$\bullet$ PIN & $3 \%$ \\
\hline$\bullet$ Vesícula seminal & $3 \%$ \\
\hline$\bullet$ Cáncer de próstata & $1,5 \%$ \\
\hline$\bullet$ Esferolito residual & $1,5 \%$ \\
\hline$\bullet$ Prostatitis aguda & $1,5 \%$ \\
\hline
\end{tabular}


TABLA III. HISTOLOGÍA DE LA ZONA CERVICOCAPSULAR RESECADA.

\begin{tabular}{|l|l|}
\hline$\bullet$ Músculo liso y fibrosis & $66 \%$ \\
\hline$\bullet$ Sólo músculatura lisa & $17 \%$ \\
\hline$\bullet$ Sólo fibrosis & $6 \%$ \\
\hline$\bullet$ Tejido fibroadiposo & $4,2 \%$ \\
\hline
\end{tabular}

una medida tendente a disminuir o anular los espasmos postoperatorios de los pacientes intervenidos al eliminar una zona especialmente rica en musculatura y alfa receptores. La verdad es que su aplicación ha supuesto la disminución notable de tal enojoso efecto en el postoperatorio inmediato.

d) Datos de piezas de cirugías radicales de próstata y veiiga: Se han examinado, a modo de control, las secciones histológicas correspondientes al tejido prostático de sujetos entre 51 y 57 años sometidos a cirugía radical de próstata o vejiga con el fin de evaluar los cambios de las zonas de transición y periférica al objeto de su comparación con los obtenidos en las cirugías del presente estudio por HBP. Los hallazgos de la zona transicional consistieron en la presencia de pequeños nódulos hiperplásicos predominantemente glandulares en grados variables, entre leves y moderados, con escasos fenómenos de quistificación secundaria y compactación de las glándulas
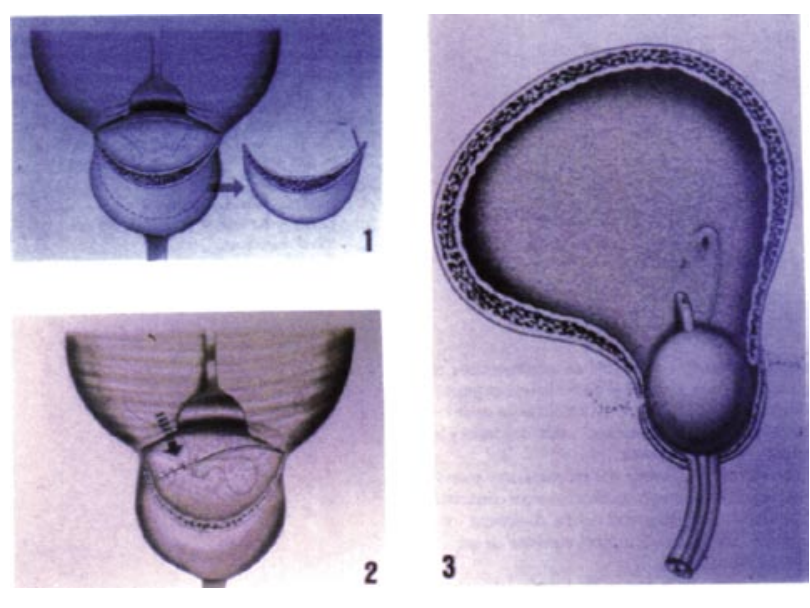

3

FIGURA 9. En nuestra técnica el globo vesical queda incluido en la loge lo que facilita el cálculo del volumen residual postquirúrgico. moderada sin correlación exacta con la edad de los sujetos control.

Por otro lado, los cambios en la zona periférica son proporcionales a la intensidad del aumento de volumen de la zona transicional. Así, cuando el grado de hiperplasia es leve, la zona periférica muestra las características estructuras glandulares ramificadas rodeadas de estroma y asociadas a componente inflamatorio muy leve. En el otro extremo, cuando la hiperplasia transicional es más acusada, dichas glándulas presentan un aspecto aplanado y comprimimido con reducción notable de su luz y del número de ramificaciones glandulares y un componente inflamatorio más evidente.

Hemos señalado, al comienzo, que el estudio realizado era histomorfométrico y hemos visto, hasta ahora, las lesiones histológicas encontradas. El aspecto "morfológico" del área intervenida lo obtenemos mediante ecografía transrectal al $3^{\circ}$ día del postoperatorio. Como en nuestra técnica quirúrgica colocamos siempre el globo de la sonda en la celda prostática (Figura 9), la ecografía transrectal nos da el volumen total del que luego restamos el volumen del globo para obtener el volumen basal de tejido residual que queda después de la adenomectomía (Figura 10).

En este momento disponemos de datos de los volúmenes prostáticos preoperatorios, de la pieza quirúrgica y de la loge, con globo incluido, en el inmediato postoperatorio. Conscientes de que las fórmulas empleadas para calcular los volúmenes de esferoides "orgánicos" pueden no ser exactos hemos recurrido a comprobar si existía una buena o suficiente correlación entre esos tres volúmenes considerando que la suma del volumen de la pieza junto al de la próstata residual debería aproximarse lo más posible al volumen de la ecografía preoperatoria. Realizada tal operación nos da un coeficiente de 1,03 que se aproxima bastante al "ideal teórico" del 1 . 


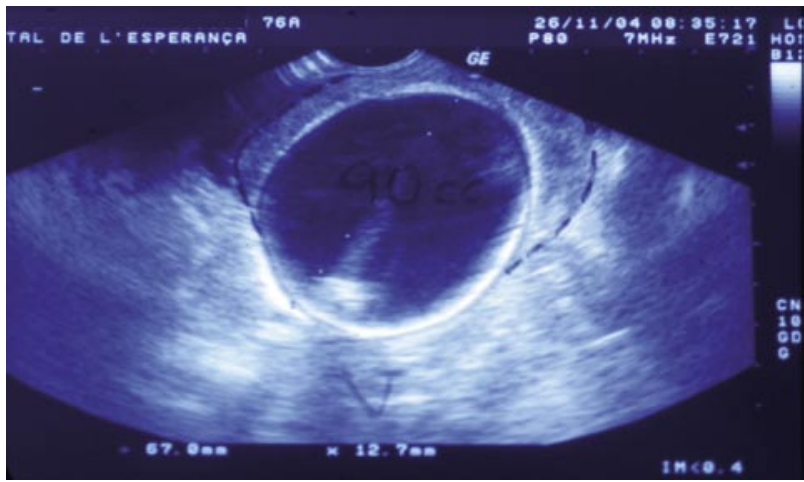

FIGURA 10. Ecografía transrectal al 3ํ día postoperatorio que permite el cálculo del volumen de próstata periférica restante conociendo el volumen del globo de la sonda.

Partiendo de esa confianza podemos obtener los datos siguientes:

1. La cirugía de la ATH "extirpa" un $76 \%$ de la glándula total por enucleación.

2. El tejido residual restante oscilará entre el $0,6 \%$ y el $38 \%$ del conocido antes de la intervención con una media cercana al $25 \%$.

3. Por tanto, aunque enucleamos el "adenoma", va a quedar tejido residual en torno a los 27 grs (valor promedio) y sobre el que puede asentarse una nueva patología prostática.

En la literatura hay buenos ejemplos de estudios mofométricos y volumétricos de las glándulas en el pre y postoperatorio relacionándolos con el peso extirpado. Así, Hastak (2) llama la atención sobre la no correspondencia entre volúmenes pre y postoperatorios que él atribuye a un "efecto esponja" del tejido glandular residual una vez ha desaparecido la compresión por el "adenoma".

En España, Espuela (3) ha realizado un importante estudio con 220 resecados y 122 adenomectomizados de próstata e introduce por primera vez el concepto de "próstata expandida" para denominar esa proliferación del tejido periférico de la glándula. El estudio es básicamente por ecografía y por análisis volumétricos aunque en 2 casos llega a practicar biopsias del tejido residual con resultado de "tejido prostático sin alteraciones" y "adenocarcinoma de próstata" que no nos dice mucho en cuanto a la explicación de lo que ha sucedido con ese tejido de la próstata caudal aunque, no cabe duda que es muy valorable el hallazgo del carcinoma. Estudios similares (4-7) abundan en el mismo sentido pero no aportan datos sobre la evolución histológica.

\section{COMENTARIO}

La sucesión de los hechos que nos han impulsado a iniciar este estudio ha sido la siguiente:

1) Comprobación de que tras una adenomectomía prostática puede llegar a reproducirse una neopatología obstructiva bien por retracción o por el desarrollo de una neohiperplasia.

2) Constatación históricamente conocida que la próstata residual puede ser asiento de patologia neoplásica

3) Ausencia de trabajos que se refieran al potencial de desarrollo hiperplásico de la próstata residual.

Sobre estas premisas hemos sentado nuestra hipótesis que pretende averiguar si esa evolución puede ser previsible en función de las lesiones basales encontradas en el acto operatorio a contrastar con las que se obtendrán en el seguimiento entre el 1 y $2^{\circ}$ año y, en último término, con las que se obtengan ante la reproducción de clínica obstructiva que puede acaecer en el futuro.

Entendemos que existe, además, un valor añadido en este estudio como es el seguimiento de aquellos casos que, previa a la intervención, han presentado cifras anómalas de PSA entre 4 y $10 \mathrm{ng} / \mathrm{ml}$, siendo biopsiados en una o varias ocasiones con resultado negativo. Muchos de tales casos no han presentado lesiones neoplasias en la pieza enucleada por lo que las biopsias posteriores de esa zona residual restante, aún con PSA normales, pueden tener un evidente interés.

\section{CONCLUSIÓN}

Nuestra intención, en este primer artículo, es exponer los hallazgos histomorfométricos que pudiéramos llamar "basales" y que, seguramente, se van a ver alterados con el paso del tiempo. En este sentido tenemos iniciado ya el estudio de los cambios histológicos a corto medio plazo mediante biopsias con resector de la "próstata expandida" junto con los oportunos estudios ecográficos a comparar con los que al día de hoy conocemos. 


\section{BIBLIOGRAFÍA y LECTURAS RECOMENDADAS (*lectura de interés $y^{* *}$ lectura fundamental)}

*1. BALLESTEROS, J.J.: "La vesicocapsuloplastia: Variante técnica en la cirugía retropúbica por HBP. Informe preliminar". Arch. Esp. Urol., 56: 1033, 2003.

*2. HASTAK, S.M.; GAMMELGAARD, J.; HOLM, H.H.: "Transrectal ultrasonic volume determination of the prostate. A preoperative and postoperative study". J. Urol, 127: 1115, 1982.

*3. ESPUELA, R.; ABAD, F.; HOGUERAS, M.A., y cols.: "Ecografía postoperatoria del hueco prostático: Próstata expandida". Arch. Esp. Urol., 41: 831, 1988.

*4. CASTIÑEIRAS, J.; RODRIGUEZ RUBIO, F.; LÓPEZ, A. y cols.: "Anatomía ecográfica de la glándula prostática hiperplásica: Correlación anatomo ecográfica". Temas Monográficos en Urología, 1: 3, 1989.

5. OSCA, J.M.; BORONAT, F.; DOMINGUEZ, C. y cols.: "La ecografía abdominal en la determinación del peso prostático". Actas Urol. Esp., 16: 305, 1992.

6. PASSAS, J.; RODRIGUEZ, A.; LEIVA, O.: "Correlaciones volumétricas del adenoma prostático mediante ecogrfía transrectal". Actas Urol. Esp., 18: 365, 1994.

7. AARNINK, R.G.; HUYNEN, A.L.; GIESEN, R.J.B. y cols.: "Automated prostate volume determination with ultrasonographyc imaging". J. Urol., $153,1995$. 